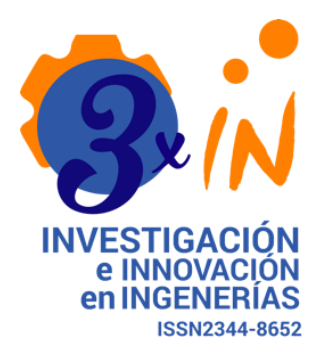

Open Access

Recibido:

5 junio de 2019

Aceptado:

9 septiembre de 2019

Publicado:

1 enero de 2020

Correspondencia:

dlopezj@uniguajira.edu.co adhenriquezj@uniguajira.edu.co

DOI:

https://doi.org/10.17081/invinno.8.1 .3535

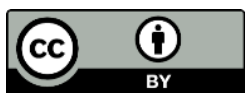

(c) Copyright: Investigación e Innovación en Ingenierías

\section{Transferencia tecnológica para la producción limpia en la minería de materiales aluviales en La Guajira, Colombia}

\section{Technology transfer for clean production in mining of alluvial mate- rials in La Guajira, Colombia}

\author{
Kevin Polo Bornachera (iD), Danny Daniel López Juvinao \\ Alfonso Henríquez Jaramillo iD \\ Universidad de La Guajira, Colombia
}

Resumen

Objetivo: Analizar la transferencia tecnológica para la producción limpia en la minería de materiales aluviales en La Guajira, Colombia. Metodología: La investigación fue de tipo descriptiva, con diseño no experimental, transeccional y de campo. La población estuvo conformada por tres empresas: Agregados Río Negro, Valores y Contratos S.A. "Valorcon S.A." y La Macuira Inversiones y Construcciones S.A, que conforman el sector de la minería de materiales aluviales en La Guajira. Para la recolección de datos se utilizó como técnica la encuesta, empleándose como instrumento dirigido a los informantes, con preguntas cerradas y cinco alternativas de respuesta, constituido por 49 ítems. Se aplicó la validez de criterio con expertos, luego se realizó el cálculo de la confiabilidad a través del coeficiente Alfa de Cronbach, obteniéndose un valor de 0,90. Resultados: Se encontró que la mayor parte de la población realiza la transferencia directamente con el proveedor de la tecnología, es decir solo intervienen un receptor y quien provee, la población estudiada a veces asiste a ferias y congresos para obtener información sobre nuevas tecnologías, y entre las barreras se destaca que gran parte de la población no utiliza tecnologías adecuadas para la producción limpia. Conclusiones: La transferencia de tecnología en las empresas estudiadas estuvo enmarcada por las carencias de tecnologías que propicien una producción limpia, el factor competitivo de la tecnología y aumento de la calidad de los proyectos.

Palabras claves: Producción Limpia, Elementos, Transferencia Tecnológica, Minería, Materiales Aluviales.

\begin{abstract}
Objective: To analyze the technological transfer for clean production in the mining of alluvial materials in La Guajira, Colombia. Methodology: The research was descriptive, with a nonexperimental, transectional and field design. The population was made up of three companies: Agregados Río Negro, Valores y Contratos S.A. "Valorcon S.A." and La Macuira Inversiones y Construcciones S.A, which make up the alluvial materials mining sector in La Guajira. For the data collection, the survey was used as a technique, using as an instrument aimed at the informants, with closed questions and five response alternatives, consisting of 49 items. The criterion validity was applied with experts, then the reliability calculation was made through Cronbach's alpha coefficient, obtaining a value of 0.90 . Results: It was found that most of the population makes the transfer directly with the technology provider, that is, only one receiver is involved and who provides, the population studied sometimes attends fairs and congresses to obtain information on new technologies, and Among the barriers, it is noted that a large part of the population does not use adequate technologies for clean production. Conclusions: The transfer of technology in the companies studied was framed by the lack of technologies that lead to clean production, the competitive factor of technology and increased quality of projects.
\end{abstract}

Keywords: Clean Production, Elements, Technological Transfer, Mining, Alluvial Materials.

Como citar (IEEE): K. Polo Bornachera, D. López Juvinao., y A. Henríquez Jaramillo, “Transferencia tecnológica para la producción limpia en la minería de materiales aluviales en La Guajira, Colombia", Investigación e Innovación en Ingenierías, vol. 8, nº. 1, 2020. DOI: https://doi.org/10.17081/invinno.8.1.3535 


\section{Introducción}

La dinámica del desarrollo, signada por la recurrencia a las tecnologías de la información acentúa los procesos de cambio y puntualiza la necesidad de una nueva proyección en la gestión de la ciencia, la tecnología, la innovación y el medioambiente y, con ellas, en la gestión del conocimiento. Esto permite que se considere al conocimiento como un recurso estratégico que está presente en cada proceso de transferencia que se realice. Los mecanismos de cambio que se están operando en las tecnologías, movidos en su mayoría por fines económicos a partir del síndrome del consumismo, obligan a los países menos desarrollados a replantearse el proceso de transferencia tecnológica. Cada espacio de tiempo juega un papel fundamental en su economía de escala, haciendo que para dichos países el integrar la gestión de las dimensiones antes referidas resulte un principio fundamental en el enfoque de una gestión empresarial sostenible [1].

En efecto, la transformación de la naturaleza por parte del ser humano es tan antigua como este. No se entendería de otra manera todos los beneficios en los nuevos productos y tecnologías que hoy se tienen para satisfacer los requerimientos de la vida diaria. Basta que miremos a nuestro alrededor para darnos cuenta de la cantidad de cosas fabricadas por la especie humana que facilitan el pasar cotidiano [2].

Verdaderamente, resulta interesante observar que, en los últimos tiempos, se está generando una dinámica orientada a fomentar la capacidad de aprendizaje y de conocimiento en las organizaciones como uno de los elementos críticos para mantener a la organización en un estado de desarrollo y evolución permanente. De hecho, la experiencia muestra claramente cómo aquellas organizaciones que no han desarrollado una capacidad de aprendizaje han visto reducida su capacidad competitiva. Incluso se ha reconocido que el aprendizaje es un comportamiento inherente a toda organización, destinado a mejorar su capacidad de adaptación y anticipación a las exigencias del entorno, así como a dotarla de una idiosincrasia propia y difícil de imitar fuera de su contexto [3].

Dentro de este marco, a menudo se plantea el argumento de que el concepto de desarrollo sustentable es incompatible con la industria extractiva de minerales y que la minería en cualquier forma no es sustentable. La tecnología que se genera a partir de la interacción eficiente y efectiva de los sectores permite legitimar la soberanía nacional y generar continuamente conocimiento para lograr el progreso social y tecnológico [4]. Entendiéndose por eficiencia el logro de los objetivos establecidos a partir del mínimo uso de los recursos utilizados y por efectividad el impacto social resultante de los proyectos tecnológicos [5]. 
Ahora bien, en Colombia, La Guajira es un departamento caracterizado por la riqueza mineral que alberga, tales como materiales aluviales, carbón, oro, sal, entre muchos otros, por esta razón son numerosas las explotaciones mineras presentes en esta región de Colombia, por lo cual no es ajena a la generación de grandes ingresos económicos a causa de la actividad, además de fuentes de empleo a gran parte de su población.

Ciertamente, hoy existen tecnologías que permiten manejar una buena gestión ambiental en una empresa minera, particularmente si se quiere atacar los problemas en las causas que los generan y no en las consecuencias finales. Sin embargo, dado que atacar los problemas en su raíz requiere inversiones de dinero mayores, las empresas con operaciones mineras en La Guajira optan por atacar solo los efectos finales, haciendo por ejemplo una simple neutralización y disposición final de los efluentes, lo que no soluciona en su totalidad el problema ambiental y puede causar, según el manejo, daños incluso mayores, por lo que de la transferencia de tecnologías de producción más limpia adecuadas depende una buena gestión [2].

Aunado a lo descrito anteriormente, en la minería de materiales aluviales legalmente constituida en este departamento, aunque hay tecnificación, esta se basa solo en tratar los impactos vertidos al medio; por el contrario, con las tecnologías de producción limpias se generaría una potencial protección del medio en el momento mismo de la extracción en la operación minera, lo que llevaría a vertimientos al ambiente tratados y con mejores perjuicios.

Lo anterior sería lo ideal, pero en el departamento de La Guajira no se implementan las tecnologías acordes, aunque se presume que dentro del paquete tecnológico transferido - generalmente de otros países, en los que la legislación ambiental es rígida y ha obligado a un mejoramiento de las tecnologías convencionales a las de producción limpia- viene incluido el paquete ambiental, que las empresas no toman en cuenta, porque desconocen la legislación o simplemente no ahondan en el tema ambiental y dejan a un lado la responsabilidad social ambiental para disminuir costos.

Particularmente, la correcta transferencia de tecnologías de producción limpia en la minería de materiales aluviales en La Guajira es garantía para un buen proceso extractivo con eficiencia y responsabilidad ambiental, pero se debe determinar por qué las tecnologías implementadas aún siguen causando daños al medio a pesar de los avances mundiales en este campo y con tecnologías que son producidas en países con una responsabilidad establecida.

Teóricamente, la transferencia tecnológica es, en esencia, el movimiento de tecnología y/o conocimiento, un valioso activo desde el punto de vista socioeconómico que puede incluir tanto medios técnicos como el conocimiento asociado (saber hacer y experiencia), desde un proveedor (universidad, organismo de investigación, centro tecnológico, empresa...), 
que la comercializa, hacia un receptor (generalmente empresa), que la adquiere a cambio de una contraprestación habitualmente económica [6]. También, la definen como ventas o concesiones, hechas con ánimo lucrativo, de tecnología que deben permitir al licenciatario o comprador fabricar en las mismas condiciones que el licenciante o vendedor [7].

Por su lado, la transferencia tecnológica es un proceso lineal donde se observan claramente sus distintas fases, que van desde su proveedor hasta su receptor, caracterizadas por la identificación, búsqueda, selección, evaluación, absorción de la tecnología, tomando en cuenta elementos y aspectos para que el proceso de transferencia tecnológica se dé en la organización [8].

A su vez, la transferencia tecnológica es la transmisión del saber hacer y de conocimientos tecnológicos, con la finalidad de desarrollar nuevas aplicaciones, transformándose en un factor determinante para el proceso de innovación y competitividad [9]. La variable del presente estudio también puede ser definida como el proceso que se encarga de la transmisión de conocimiento, que permiten a la empresa receptora desarrollar ventajas competitivas y obtener superioridad relativa con respecto a otras empresas del mercado, donde incorpora la dimensión de mecanismos, tipos y a su vez establece indicadores [10].

De igual manera, la transferencia tecnológica forma parte del proceso de innovación de cualquier empresa, la cual requiere perfeccionar los medios de como seleccionar, adquirir, adaptar y adoptar, para la valorización de sus procesos [11]. Finalmente, la transferencia tecnológica se concibe como una serie de procedimientos que intenta insertar los elementos de innovación para aumentar la producción en cualquier organización, independientemente de su naturaleza [12].

De acuerdo con lo anterior, la transferencia de tecnología es el pilar innovativo de toda empresa, que busca aumentar su productividad y mantenerse a la vanguardia en el mercado actual, seleccionando, adquiriendo, adaptando y poniendo en marcha la tecnología obtenida acorde con las necesidades propias de la actividad a realizar.

\section{Metodología}

Esta investigación fue de tipo descriptiva [13], con un diseño no experimental, transeccional y de campo [14]. La población de este estudio fue definida como finita, dado que está relacionada con las empresas dedicadas a la minería de materiales aluviales y su transferencia tecnológica de producción limpia, representada en tres (3) empresas dentro del departamento de La Guajira: Agregados Río Negro, Valores y Contratos S. A. "Valorcon S. A." y La Macuira inversiones y construcciones S. A. [15]. 
Cada empresa que constituye la población cuenta con informantes claves, entre los cuales se destacan la gerencia, los administradores y el área de compra y transferencia de tecnologías propias de la labor extractiva (coordinador de compras), representante ambiental, representante minero, además de operadores, para un total de 24 informantes (Tabla 1), de quienes se obtuvo la información que sirvió de base para el análisis y conclusiones de la investigación.

Tabla 1. Informantes clave

\begin{tabular}{|c|c|}
\hline Sujetos & Cantidad \\
\hline Gerente & 3 \\
\hline Administrador & 3 \\
\hline Coordinación de compras & 3 \\
\hline Representante ambiental & 3 \\
\hline Representante minero & 3 \\
\hline Operadores & 9 \\
\hline Total & 24 \\
\hline
\end{tabular}

Fuente: Elaboración propia

La técnica de recolección de datos utilizada en esta investigación fue la encuesta en la modalidad de cuestionario [13]. Se construyó un cuestionario tipo Likert que consiste en un conjunto de ítems presentados en forma de afirmaciones o juicios, ante los cuales se pide la reacción de los participantes. Es decir, se presenta cada afirmación y se solicita al sujeto que externe su reacción eligiendo uno de los cinco puntos o categorías de la escala.

El cuestionario se estructuró en 49 ítems, en que las opciones de respuestas fueron categorizadas con cinco posibilidades: siempre, casi siempre, a veces, casi nunca y nunca.

Para efectos de esta investigación, se tuvo en cuenta la validez de contenido, realizado mediante la consulta técnica a expertos en las áreas de metodología de la investigación, transferencia de tecnología, minería de producción limpia, buscando una validación del instrumento, en relación con su contenido, efectividad, coherencia, entre otros aspectos.

Igualmente se midió la confiabilidad, para lo cual se realizó una prueba piloto, en empresas de características similares, obteniendo una confiabilidad de 0,90 , lo que indica que el instrumento es de muy alta confiabilidad, por lo cual son completamente confiables los datos que se obtuvieron en su implementación.

Así mismo, se utilizó la media aritmética, la desviación estándar, frecuencias acumuladas y relativas, que permitieron la categorización de ítems, en relación con analizar los procesos relacionados con los elementos en la transferencia tecnológica de producción limpia del estudio. Asimismo, en el análisis estadístico de la información se utilizó un computador de escritorio 
marca Dell y el paquete estadístico Excel 2016 para Windows. A continuación, se muestra el Tabla 2 identificando como baremo para el estudio de la media.

Tabla 2. Baremo para el estudio de la media

\begin{tabular}{|c|c|c|}
\hline Alternativas & Límites & Categoría \\
\hline Siempre & $4.21 \geq \mathrm{X}<5.00$ & Muy alta presencia del indicador \\
\hline Casi siempre & $3.41 \geq \mathrm{X}<4.20$ & Alta presencia del indicador \\
\hline A veces & $2.61 \geq \mathrm{X}<3.40$ & Moderada presencia del indicador \\
\hline Casi nunca & $1.81 \geq \mathrm{X}<2.60$ & Baja presencia del indicador \\
\hline Nunca & $1.00 \geq \mathrm{X}<1.80$ & Muy baja presencia del indicador \\
\hline
\end{tabular}

Fuente: elaboración propia

\section{Resultados y discusión}

De acuerdo con la Tabla 1, la dimensión elementos en la transferencia tecnológica obtuvo una media de 3,6, lo que indica que hay una alta presencia de los indicadores (Tabla 2 ) en las organizaciones dedicadas a la minería de materiales aluviales. La desviación arrojó un promedio de 1,1, lo que indica una muy alta dispersión. De igual forma, se observa que el indicador actores obtuvo una media de 3,6 presentando una alta presencia, donde se evidencia que el $39,2 \%$ de la población casi siempre realiza la transferencia tecnológica, estudiando sus necesidades, utilizando empresas que provean la tecnología acorde y especificando el nivel técnico que se requiere para la operación.

Tabla 3. Elementos en la transferencia tecnológica

\begin{tabular}{|c|c|c|c|c|c|c|c|c|c|}
\hline \multicolumn{7}{|c|}{ Dimensión: elementos en la transferencia tecnológica } \\
\hline Indicador & Media & Desviación & Frecuencias & \multicolumn{5}{|c|}{ Opciones de respuesta } & Total \\
\cline { 5 - 8 } & & & & $\mathrm{S}$ & $\mathrm{cs}$ & $\mathrm{av}$ & $\mathrm{cn}$ & $\mathrm{N}$ & \\
\hline Actores & 3,6 & 1,1 & $\mathrm{fa}$ & 5 & 9 & 5 & 3 & 2 & 24 \\
\cline { 5 - 9 } & & & $\mathrm{fr}(\%)$ & 21,7 & 39,2 & 21,7 & 10,8 & 6,7 & 100 \\
\hline Modalidades & 3,2 & 1,2 & $\mathrm{fa}$ & 4 & 6 & 6 & 6 & 2 & 24 \\
\cline { 4 - 8 } & & & $\mathrm{fr}(\%)$ & 16,1 & 26,8 & 24,4 & 26,2 & 6,5 & 100 \\
\hline Motivaciones & 4,2 & 0,92 & $\mathrm{fa}$ & 11 & 7 & 5 & 1 & 0 & 24 \\
\cline { 4 - 8 } & & & $\mathrm{fr}(\%)$ & 46,5 & 29,2 & 18,8 & 5,6 & 0,0 & 100 \\
\hline Promedio & 3,6 & 1,1 & & & & & & & \\
\hline
\end{tabular}

Fuente: Elaboración propia

Además, la Tabla 3 muestra que para el indicador modalidades se obtuvo una media de 3,2, que de acuerdo con el baremo de análisis (Tabla 2) indica que hay una moderada presencia, por lo cual un $26,8 \%$ de la población indica que casi siempre las tecnologías son recibidas directamente del proveedor $y$ mediante un contrato escrito entre las partes que gobiernan la transferencia. Cabe destacar que se obtuvo un porcentaje de $26,2 \%$ en la 
opción casi nunca, donde se relaciona que la transferencia en muy pocas ocasiones se hace mediante licenciamiento y se obtiene mediante un intermediario sin tener contacto con quien provee la tecnología.

Con respecto a las motivaciones, la Tabla 3 indica que obtuvo una media de 4,2 , lo que se traduce en alta presencia del indicador, cercano a la muy alta presencia, donde el mayor porcentaje encontrado fue 46,5 para la opción de respuesta siempre, lo que indica que existen carencias para la producción limpia, el factor competitivo de la tecnología, se aumenta la calidad de los proyectos y que estos sean acordes con la normativa ambiental vigente, lo que genera motivaciones para transferir tecnologías de producción limpia.

Para el caso del indicador actores, se observa que los resultados coinciden con el postulado de González [6], quien menciona que se trata de las partes, operadores o agentes involucrados en el proceso de transferencia de tecnología (proveedor de la tecnología, receptor de la tecnología, intermediario del proceso). Se encontró que la mayor parte de la población realiza la transferencia directamente con el proveedor de la tecnología, es decir, solo intervienen un receptor y quien provee; no existe ningún tipo de intermediario que acelere, facilite o asesore el proceso de transferencia.

En el indicador modalidades, coinciden las propuestas por González [6], entre las que plantea que esta se puede dar según el mecanismo de transferencia, según la vía de la transferencia, según la formalidad de la colaboración y según el enfoque de la transferencia. De acuerdo con lo anterior, según el mecanismo de transferencia, esta generalmente se produce por un contrato escrito entre las partes, generalmente no existe licenciamiento; según la vía, está enmarcada de tipo directa, ya que se desarrolla con el proveedor sin la inclusión o apoyo de algún intermediario. Se dan las transferencias de tipo formal, y de acuerdo con el enfoque de ambas partes de la transferencia (proveedor y receptor), debido a que se evaluaron las necesidades propias de la empresa y ofrecimientos de las organizaciones que suministran la tecnología.

Para el caso de las motivaciones, que de acuerdo con González [6] son las razones, causas o factores que originan, impulsan y/o condicionan la transferencia de tecnología, las cuales pueden tener diferentes dimensiones, estas estuvieron motivadas por las carencias de tecnologías que propiciaran una producción limpia, el factor competitivo de la tecnología, el aumento de la calidad de los proyectos y que estos sean acordes con la normativa ambiental vigente. 
Tabla 4. Proceso de transferencia tecnológica

\begin{tabular}{|c|c|c|c|c|c|c|c|c|c|}
\hline \multicolumn{10}{|c|}{ Dimensión: proceso de transferencia tecnológica } \\
\hline \multirow[t]{2}{*}{ Indicador } & \multirow[t]{2}{*}{ Media } & \multirow[t]{2}{*}{ Desviación } & \multirow[t]{2}{*}{ Frecuencias } & \multicolumn{5}{|c|}{ Opciones de respuesta } & \multirow[b]{2}{*}{ Total } \\
\hline & & & & $\mathrm{S}$ & cS & av & $\mathrm{cn}$ & $\mathrm{N}$ & \\
\hline \multirow{2}{*}{$\begin{array}{c}\text { Búsqueda de la } \\
\text { tecnología }\end{array}$} & 3,3 & 0,8 & fa & 2 & 7 & 13 & 3 & 0 & 24 \\
\hline & & & $\mathrm{fr}(\%)$ & 6,5 & 28,0 & 54,2 & 11,3 & 0,0 & 100 \\
\hline \multirow{2}{*}{ Evaluación } & 4,2 & 0,7 & $\mathrm{fa}$ & 9 & 12 & 3 & 1 & 0 & 24 \\
\hline & & & fr (\%) & 38,2 & 47,9 & 11,1 & 2,8 & 0,0 & 100 \\
\hline \multirow[t]{2}{*}{ Negociación } & 3,6 & 0,8 & fa & 3 & 10 & 10 & 1 & 0 & 24 \\
\hline & & & fr (\%) & 11,1 & 41,7 & 41,7 & 5,6 & 0,0 & 100 \\
\hline \multirow[t]{2}{*}{ Adquisición } & 4,0 & 0,8 & fa & 8 & 9 & 7 & 0 & 0 & 24 \\
\hline & & & fr (\%) & 33,3 & 35,4 & 31,3 & 0,0 & 0,0 & 100 \\
\hline \multirow[t]{2}{*}{ Adaptación } & 3,3 & 0,9 & fa & 2 & 9 & 9 & 5 & 0 & 24 \\
\hline & & & fr (\%) & 6,3 & 37,5 & 35,4 & 20,8 & 0,0 & 100 \\
\hline Promedio & 3,7 & 0,8 & & & & & & & \\
\hline
\end{tabular}

Fuente: Elaboración propia

De acuerdo con la Tabla 4, la dimensión proceso de transferencia tecnológica arrojó una media de 3,7 , lo que indica alta presencia de los indicadores; en el caso de la desviación, este arrojó un promedio de 0,8 , lo que indica una alta dispersión. Así mismo, el indicador búsqueda de la tecnología arrojó una media de 3,3, que indica una moderada presencia del indicador con un porcentaje de $52,2 \%$, indicando que a veces se asiste a ferias y congresos para obtener información sobre nuevas tecnologías, se buscan ofertas en portales web, revistas especializadas, entre otros, además de que cuentan con sistemas formales para transferir tecnología.

De igual forma, el indicador evaluación arrojó una media de 4,2, estando en el límite entre alta y muy alta presencia en la población, con un $47,9 \%$, que coincide en que casi siempre, al momento de transferir tecnología, se evalúan parámetros tales como el costo de mantenimiento, si el tipo de tecnología convencional o de producción limpia maneja mayor o menor costo, así mismo la calidad de la transferencia y por ende el producto, si aumenta la calidad y eficiencia de la producción, evaluando tecnologías convencionales con las de producción limpia, buscando con ello el mayor beneficio para la organización.

Con respecto al indicador negociación, arrojó una media de 3,6, lo que indica una alta presencia del indicador, destacando el porcentaje $41,7 \%$ en las opciones de respuesta casi siempre y a veces con iguales resultados, donde en la mayoría de la población encuestada maneja información como elemento de negociación, para lograr aspectos beneficiosos entre las partes, que permitan mejoras en los procesos de producción limpia.

En el caso del indicador adquisición, este arrojó una media de 4,0, dando como resultado un porcentaje de $35,4 \%$, en que la población de estudio casi 
siempre considera importante la acumulación de capacidades tecnológicas, el uso adecuado de las tecnologías, el orientar el uso de las tecnologías en procesos de aprendizaje, y la modificación en algunos casos de la tecnología como factor clave en los procesos de transferencia. De igual forma, es importante destacar que las opciones de respuesta siempre y a veces obtuvieron porcentajes de 33,3 y $31,3 \%$, respectivamente, muy cercanos al valor más alto registrado, por lo que se presume que al tener diversas características las organizaciones, a pesar de pertenecer al mismo sector productivo, difirieren en algunos aspectos propios de este indicador. El indicador adaptación es el último perteneciente a esta dimensión, el cual arrojó una media de 3,3, lo que indica que se encuentra con una moderada presencia entre la población consultada, reflejado en que el $37,5 \%$ y el $35,4 \%$ pertenecientes a casi siempre y a veces respectivamente se presentó con resultados bastante cercanos, lo que indica que por las características de las diversas organizaciones casi siempre o a veces realizan cambios a los procesos para incorporar la producción limpia a los sistemas existentes, así como también cuentan en muchos casos con personal que está capacitado para adaptar las necesidades propias de la organización a las tecnologías adquiridas en busca de una producción más limpia.

Para el indicador búsqueda de tecnología, se encontró que la población estudiada a veces asiste a ferias y congresos para obtener información sobre nuevas tecnologías, se buscan ofertas en portales web, revistas especializada, entre otros, además de que cuentan con sistemas formales para transferir tecnología, lo que coincide con el postulado de Velásquez [16], quien menciona que si el proveedor de la tecnología se ubica fuera del país, entonces hay que localizarlo por medios diversos: Internet, búsqueda de patentes, directorios empresariales, revistas especializadas, catálogos, ferias y exposiciones, etc., que es lo que usualmente sucede, porque la transferencia en estas organizaciones generalmente se realiza con proveedores internacionales.

Para el caso del indicador evaluación, se presentó que las organizaciones en la mayoría de los casos evalúan parámetros tales como el costo de mantenimiento, si el tipo de tecnología convencional o de producción limpia maneja mayor o menor costo, así mismo la calidad de la transferencia y por ende el producto, si aumenta la calidad y eficiencia de la producción, evaluando tecnologías convencionales con las de producción limpia, que coincide con lo propuesto por Velázquez [16], quien establece parámetros como definir los criterios de evaluación por utilizar, examinar la información técnica, valorar la información económica y verificar las principales ventajas y desventajas, aspectos tenidos en cuenta por la población.

Con respecto al indicador negociación, esta estuvo dominada por elementos tales como que manejan información como elemento de negociación, para lograr aspectos beneficiosos entre las partes, que permitan mejoras en los procesos de producción limpia. Esto coincide totalmente con lo señalado por 
la Onudi en su Manual para Negociaciones de Transferencia de Tecnología, en lo referido a que el acuerdo de transferencia de tecnología que conduce a una relación satisfactoria a largo plazo entre dos o más partes es uno en que las partes reconocen que debe proporcionar beneficios a todas las partes, que es lo que buscan las organizaciones con el proceso de transferencia.

Para el indicador adquisición, se considera importante la acumulación de capacidades tecnológicas, el uso adecuado de las tecnologías, el orientar el uso de las tecnologías en procesos de aprendizaje, y la modificación en algunos casos de la tecnología como factor clave en los procesos de transferencia, aunque los resultados fueron muy cercanos a varias opciones de respuesta, lo que deja opiniones divididas con respecto a este indicador por parte de la población, lo que refleja la posición de Velázquez [16], en lo referido a las orientaciones que presenta cada organización de acuerdo con el uso que le da a cada tecnología.

Por último, en el caso del indicador adaptación, este mostró que realizan cambios a los procesos para incorporar la producción limpia a los sistemas existentes, así como también cuentan en muchos casos con personal que está capacitado para adaptar las necesidades propias de la organización a las tecnologías adquiridas en busca de una producción más limpia. Esto coindice con la posición de Velásquez [16], cuando menciona que se habla de adaptación cuando se requiere cambiar el diseño y características de una tecnología que ha sido desarrollada para operar en condiciones muy distintas a las de la región donde se va a utilizar la tecnología que se adquiere, justificado en que mucha de la tecnología convencional se ha adaptado hacia la producción limpia.

Tabla 5. Barreras para la transferencia tecnológica

\begin{tabular}{|c|c|c|c|c|c|c|c|c|c|}
\hline \multicolumn{10}{|c|}{ Dimensión: barreras para la transferencia tecnológica } \\
\hline \multirow[t]{2}{*}{ Indicador } & \multirow[t]{2}{*}{ Media } & \multirow[t]{2}{*}{ Desviación } & \multirow[t]{2}{*}{ Frecuencias } & \multicolumn{5}{|c|}{ Opciones de respuesta } & \multirow[t]{2}{*}{ Total } \\
\hline & & & & $S$ & CS & $\mathrm{AV}$ & $\mathrm{CN}$ & $\mathrm{N}$ & \\
\hline \multirow{2}{*}{$\begin{array}{c}\text { Barreras } \\
\text { tecnológicas }\end{array}$} & 3,6 & 1,2 & fa & 8 & 5 & 6 & 6 & 0 & 24 \\
\hline & & & & & & & & & \\
\hline & & & fr $(\%)$ & 31,3 & 20,8 & 22,9 & 25,0 & 0,0 & 100 \\
\hline \multirow{2}{*}{$\begin{array}{c}\text { Barreras } \\
\text { organizativas }\end{array}$} & 3,8 & 0,9 & fa & 6 & 9 & 8 & 1 & 0 & 24 \\
\hline & & & & & & & & & \\
\hline \multirow{2}{*}{ Barreras personales } & & & fr (\%) & 25,0 & 37,5 & 33,3 & 4,2 & 0,0 & 100 \\
\hline & 3,1 & 0,7 & fa & 0 & 7 & 15 & 3 & 1 & 24 \\
\hline \multicolumn{3}{|c|}{ 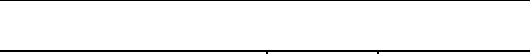 } & fr $(\%)$ & 0,0 & 27,1 & 60,4 & 10,4 & 2,1 & 100 \\
\hline Promedio & 3,5 & 0,9 & & & & & & & \\
\hline
\end{tabular}

Fuente: Elaboración propia 
Para el caso de las barreras existentes para la transferencia tecnológica, de acuerdo con la Tabla 5, esta dimensión arrojó una media de 3,6, encontrándose con una alta presencia entre la población de estudio, así mismo en la desviación estándar arrojó un promedio de 0,9 , lo que indica, de acuerdo con el baremo de análisis, que este se encuentra en la categoría de muy alta dispersión.

El indicador barreras tecnológicas obtuvo una media de 3,6 con una alta presencia en la población, dando como resultado el porcentaje más alto $31,3 \%$, perteneciente a la categoría siempre, y $25,0 \%$ para la siguiente, perteneciente a la opción de respuesta casi nunca, lo que representa un elemento diferenciador de estas organizaciones, algunas de las cuales utilizan tecnologías adecuadas para generar procesos de producción limpia, dando como resultado mayor eficiencia en los procesos al incorporarlos, mientras que otra gran parte de la población casi nunca lo hace.

Así mismo, para el indicador barreras organizativas se obtuvo una media de 3,8 , lo que la deja con una alta presencia entre la población de estudio, con un mayor porcentaje para la opción de respuesta casi siempre con un $37,5 \%$, seguido por a veces con un $33,3 \%$, reflejados en que una parte de la población realiza la planificación correspondiente a los procesos de transferencia tecnológica, se comunica con los miembros de la empresa acerca del tipo de transferencia y un control técnico correspondiente al proceso, mientras que otra parte de la población lo hace solo algunas veces.

Con respecto al indicador barreras personales, este manifestó una media de 3,1 , ubicándola en una moderada presencia dentro de la población estudiada como se indica en la Tabla 3, en este caso el porcentaje de mayor relevancia obtuvo un valor de $60,4 \%$ en la categoría a veces, lo que puede indicar que en algunas ocasiones se observa resistencia hacia la incorporación de nuevas tecnologías que permitan una producción más limpia por parte de los usuarios de estas organizaciones, así mismo, que estos manifiesten rechazo al proceso de adopción de nuevas tecnologías.

El indicador barreras tecnológicas, de acuerdo con la posición de Getec, citado por Manosalva [11], que menciona que estas se enfrentan cuando la tecnología no es adecuada para los problemas que se pretenden resolver, en este caso es acorde con los resultados arrojados, debido a que solo algunas de las organizaciones utilizan siempre tecnologías adecuadas para generar procesos de producción limpia, dando como resultado mayor eficiencia en los procesos al incorporarlos, mientras que otra gran parte de la población casi nunca lo hace, por lo que se presume que gran parte de la población se topa con estas barreras debido a que la tecnología no es adecuada para un proceso de producción limpia. En el caso del indicador barreras organizativas, el comportamiento de la organización ante los cambios muestra que las empresas enfrentan mayor resistencia a los cambios organizativos, lo que coincide con los resultados obtenidos que presentan que una parte de la población realiza la planificación 
correspondiente a los procesos de transferencia tecnológica, se comunica con los miembros de la empresa acerca del tipo de transferencia y un control técnico correspondiente al proceso, mientras que otra parte de la población lo hace solo algunas veces, lo que genera que en algunos casos existan barreras a causa de la organización interna.

Para culminar, respecto al indicador barreras personales se encontró que algunas veces se observa resistencia hacia la incorporación de nuevas tecnologías que permitan una producción más limpia por parte de los usuarios de estas organizaciones, así mismo que estos manifiestan rechazo al proceso de adopción de nuevas tecnologías, lo que es acorde con la posición de Castellanos [17], quien manifiesta que son interferencias de la comunicación que provienen de las emociones, los valores y los malos hábitos del individuo, en este caso la falta de conciencia por parte de los usuarios, hacia el uso de tecnologías que contribuyan a una sostenibilidad ambiental y por ende una mejor calidad de vida.

Para el caso del objetivo general, analizar la transferencia tecnológica de producción más limpia en el proceso de la minería de materiales aluviales en el departamento de La Guajira, Colombia, todas las dimensiones obtuvieron una alta presencia, lo que indica que la variable estuvo presente y perfectamente representada en la población analizada, dando como resultado un proceso donde no se tienen en cuenta muchos aspectos (más actores, diversas motivaciones, modalidades, canales de búsqueda y evaluación, entre otros) al momento de transferir tecnología, lo que se presume que indica fallas dentro de su operación que conlleve una producción limpia.

Asimismo, indica que existen barreras que no permiten un desarrollo pleno de una operación minera con características de sostenibilidad ambiental, traducidas en carencias de tecnologías propias de la operación, fallas internas en la organización y aspectos en que los usuarios se niegan hacia nuevas tecnologías por desconocimiento y dejar de lado la importancia del medio ambiente.

\section{Conclusión}

Inicialmente, se concluyó que en la mayoría de las veces solamente se incluyen como actores el proveedor y receptor de la tecnología, dejando de lado los intermediarios como elemento menos representativo, así mismo las modalidades de transferencia se realizan de manera directa, con contratos escritos, sin que se realice licenciamiento en la mayoría de los casos como el elemento menos presente a sabiendas de las ventajas y de lo innovadoras de algunas tecnologías bajo esta modalidad. 
Por otro lado, las transferencias en la minería de materiales aluviales estuvieron motivadas por las carencias de tecnologías que propicien una producción limpia, el factor competitivo de la tecnología y aumento de la calidad de los proyectos, pero estuvo menos representada la motivación de que una producción limpia generaba valor agregado a los productos. Lo anterior se incentiva debido a que la normatividad ambiental en Colombia exige procesos de producción que deben estar a la vanguardia de las operaciones internacionales, que, si bien no siempre es así y hay fallas en la normativa, se vela por un cumplimiento que exige en gran medida la observancia de políticas de sostenibilidad.

También, se concluye que la búsqueda de tecnología se realiza en ocasiones asistiendo a ferias y congresos, buscando en portales web y revistas especializadas, puesto que generalmente se realiza la transferencia hacia países desarrollados, y los anteriores son los mecanismos que muestran las diversas tecnologías en el mercado, donde el elemento menos presente son las revisiones de revistas especializadas de centros tecnológicos sobre tecnologías de producción más limpia, dejando de lado un mecanismo importante a la hora de transferir tecnología.

Asimismo, se evalúa la tecnología a transferir bajo parámetros tales como el costo de mantenimiento, si el tipo de tecnología convencional o de producción limpia maneja mayor o menor costo, así mismo la calidad de la transferencia y por ende el producto, dejando como menos presente si las tecnologías de producción limpia aumentan la calidad y eficiencia de la producción, comparando tecnologías convencionales con las de producción limpia, dejando a estas últimas rezagadas por el costo de implementación.

Se manejan parámetros como la información para procesos de negociación que permitan un acuerdo entre las partes para realizar procesos mineros en pro de una sostenibilidad ambiental, logrando acuerdos beneficiosos, buscando una producción limpia. Los convenios son subvalorados arrojando la menor representatividad entre la población estudiada, dejando de tener en cuenta un aspecto que permitiría obtener mejoras en el proceso de producción sustentable en este tipo de minería.

La adquisición en el proceso de transferencia realiza procesos de planificación tales como la acumulación de capacidades, el uso adecuado de las tecnologías al momento de transferirlas; se presume que con esto se hacen una idea de los beneficios que ofrece cada adquisición de acuerdo con la actividad realizada. No se enfatiza en la posibilidad de adaptar tecnología al momento de adquirirla como un factor clave, dejando este apartado como el menos representativo. Por último, basados en que mucha de la tecnología es de uso convencional (no producción limpia), se realizan procesos de adaptación buscando producciones más sostenibles con la tecnología que poseen, buscando el cumplimiento de la normatividad ambiental vigente. 
De igual manera, se concluye que existen barreras tecnológicas que permitan la producción limpia dentro de las organizaciones analizadas; si bien algunas empresas implementan algunos procesos ambientalmente sostenibles, las tecnologías en su mayoría son convencionales. En el caso de las barreras organizativas, aún existen muchas falencias debido a que solo una parte de la población comunica, planifica y realiza control técnico acerca de procesos que lleven a una producción limpia.

Igualmente, existen barreras personales en las que usuarios de las tecnologías de producción limpia muestran resistencia hacia la inclusión de estas, además de que en muchas ocasiones, más que resistencia, hay rechazo, lo que no aporta para que se realicen procesos más amables con el medio ambiente, que lleven a una sostenibilidad entre lo productivo y el medio circundante. Se presume que esto sucede por los costos en que incurre la implementación y valor final del producto.

Con respecto al objetivo general de analizar la transferencia tecnológica de producción más limpia en el proceso de la minería de materiales aluviales en el departamento de La Guajira, Colombia, todo lo anterior arroja que aún son muchos los elementos que no se tienen en cuenta dentro del proceso de transferencia tecnológica realizado por estas organizaciones, lo que genera que los procesos sigan siendo mayoritariamente convencionales, dejando un poco de lado la inclusión de nuevas tecnologías y agudizando las barreras organizativas y tecnológicas dentro de la organización.

\section{Referencias bibliográficas}

1. N. Castro, "Transferencia tecnológica, la integración ciencia, innovación tecnológica y medioambiente en la empresa", Ingeniería Industrial, Vol. 35, $\mathrm{n}^{\circ}$. 3, pp. 277-288, 2014.

2. M. Sánchez, Importancia del desarrollo de tecnologías limpias para la industria del cobre. Tecnologías limpias en las industrias extractivas minero-metalúrgica y petrolera, Brasil, pp. 17-34, 2006.

3. J. Zulueta, "Gestión del conocimiento durante la transferencia tecnológica: universidad-empresa", Avanzada Científica, Vol. 13, n. 3, pp. 1-11, 2010.

4. J. Richards, "Sustainable Development and the Mineral Industry". SEG Newsletter, Society of Economic Geologists, n. 48, 2002.

5. S. Sira y R. Pérez, "Modelo Operativo de Transferencia Tecnológica para promover la interacción universitaria con los sectores externos", Revista Digital de Investigación y Postgrado, Vol. 5, n . 4, pp. 958-976, 2015.

6. J. González, Manual transferencia de tecnología y conocimiento, Segunda edición. España: The Transfer Institute, 2011.

7. P. Escorza y J. Valls, Tecnología e innovación en la empresa: dirección y gestión. México, D. F.: Alfaomega, 2003. 
8. G. Conti, "Transferencia tecnológica como instrumento estratégico en empresas mixtas del sector petrolero", Tesis doctoral, Universidad Dr. Rafael Belloso Chacín, Decanato de Investigación y Postgrado, Doctorado en Ciencias Gerenciales, Venezuela, 2013.

9. P. Barrero, "Transferencia tecnológica en el proceso de mantenimiento de equipos de explotación y transporte en el sector carbonífero del departamento de La Guajira", Tesis de Maestría, Universidad Dr. Rafael Belloso Chacín, Decanato de Investigación y Postgrado, Maestría en Gerencia de Proyectos de Investigación y Desarrollo, Venezuela, 2014.

10. A. Sánchez, "Transferencia tecnológica en las empresas de telefonía privadas ubicadas en la República Bolivariana de Venezuela", Tesis de Maestría, Universidad Dr. Rafael Belloso Chacín, Decanato de Investigación y Postgrado, Maestría en Gerencia de Proyectos de Investigación y Desarrollo, Venezuela, 2014.

11. E. Manosalva, "Transferencia tecnológica para la producción de mango tipo exportación en la ciudad de Valledupar", Tesis de Maestría, Universidad Dr. Rafael Belloso Chacín, Decanato de Investigación y Postgrado, Maestría en Gerencia de Proyectos de Investigación y Desarrollo, Venezuela, 2015.

12. M. Morales, "Transferencia tecnológica en las plantas de tratamiento de las aguas residuales en el Municipio de Valledupar Departamento del Cesar en Colombia", Tesis de Maestría, Universidad Dr. Rafael Belloso Chacín, Decanato de Investigación y Postgrado, Maestría en Gerencia de Proyectos de Investigación y Desarrollo, Venezuela, 2015.

13. R. Hernández, C. Fernández y P. Baptista, Metodología de la investigación, Quinta edición. México: Mc Graw Hill, 2010.

14. F. Arias, El proyecto de investigación, introducción a la metodología científica, Sexta edición. Venezuela: Editorial Episteme, 2012.

15. D. López, Mucho más que carbón. El escenario minero de La Guajira. Colombia: Editorial Gente Nueva, 2011.

16. G. Velázquez, Manual de trasferencia y adquisición de tecnología sostenible. Costa Rica: Editorial Cegesti, 2010.

17. O. Castellanos, Gestión tecnológica de un enfoque tradicional a la inteligencia. Colombia: Universidad Nacional de Colombia, 2007. 